\title{
Extending our tools and resources in the non-conventional industrial yeast Xanthophyllomyces dendrorhous through the application of metabolite profiling methodologies
}

\author{
Eugenio Alcalde $^{1} \cdot$ Paul D. Fraser $^{1}$
}

Received: 23 August 2017 / Accepted: 22 December 2017 / Published online: 12 February 2018

(c) The Author(s) 2018. This article is an open access publication

\begin{abstract}
Introduction Xanthophyllomyces dendrorhous is a non-conventional industrial yeast. It has the unique ability among yeasts to produce geranylgeranyl pyrophosphate derived terpenoids such as carotenoids and in particular the high value pigment astaxanthin.

Objective In order to fully exploit the industrial potential of Xanthophyllomyces using modern industrial biotechnology approaches the further development of "omic" resources in this organism are required to build on the now sequenced and annotated genome. To contribute to this goal, the present study has developed and implemented an efficient metabolite profiling system comprised of, quenching, extraction and associated GC-MS and UPLC analysis.

Method Four quenching methods and five extraction methods compatible with GC-MS and UPLC profiling were tested and validated by analysing steady state metabolite changes of Xanthophyllomyces cultivated at laboratory scale in liquid shake culture at lag, exponential and early and late stationary phases.

Results A customised Automated Mass Spectral Deconvolution and Identification System (AMDIS) library has been created for Xanthophyllomyces, over 400 compounds are present in the library of which 78 are detected and quantified routinely in polar and non-polar derived extracts. A preliminary biochemical network has been constructed. Over a standardised laboratory growth cycle, changes in metabolite levels have been determined to create reference point for future strain improvement approaches and the initial biochemical network construction. Correlation analysis has illustrated that astaxanthin formation correlates positively with different sectors of intermediary metabolism (e.g. the TCA cycle intermediates and amino acid formation), "short" saturated fatty acids and $\beta$-carotene, while other metabolites are reduced in response to astaxanthin production. These sectors of intermediary metabolism offer potential future targets for the manipulation resulting in the generation of strains with improved titres of given terpenoids.

Discussion In summary a robust metabolite profiling system for Xanthophyllomyces is in place to further our understanding and potential exploitation of this underutilised industrial yeast.
\end{abstract}

Keywords Xanthophyllomyces dendrorhous · GC-MS · Carotenoids · Metabolite profiling

Electronic supplementary material The online version of this article (https://doi.org/10.1007/s11306-017-1313-9) contains supplementary material, which is available to authorized users.

Paul D. Fraser

Paul.Fraser@rhul.ac.uk

Eugenio Alcalde

Eugenio.Alcalde@rhul.ac.uk

1 School Biological Sciences, Royal Holloway, University London, Egham Hill, Egham, Surrey TW20OEX, UK

\author{
Abbreviations \\ GC/MS Gas chromatography mass spectrometry \\ UPLC Ultra performance liquid chromatography \\ OD Optical density \\ TCA Tricarboxylic acid
}

\section{Introduction}

Xanthophyllomyces dendrorhous is a basidiomycetous yeast first reported over 40 years ago by Herman Jan Phaff and collaborators (Miller et al. 1976; Phaff et al. 1972). Xanthophyllomyces and the green alga Haematococcus pluvialis 
are the main natural sources of astaxanthin (Schmidt et al. 2011). Astaxanthin is one of the most important industrial carotenoids presently used, it is known for its reddish pink colour and is mainly used in the aquaculture feed industry. The value of the global carotenoid market in 2014 was estimated at $\$ 1.5$ billion and is expected to reach nearly $\$ 1.8$ billion in 2019 (Business Communications Company 2015). Presently, chemical synthesis remains the production method of choice despite the high cost, and unfavourable environmental credentials. However, the growing consumer demand for more natural products (Cataldo et al. 2016) and the dwindling fossil fuel reserves, from which the precursors for chemical synthesis originate, are driving the market to implement new renewable sources (Mannazzu et al. 2015). Xanthophyllomyces with its amenability to large scale mutagenesis programmes, rudimentary pathway manipulation, and improvements in culture condition have been developed in order to improve Xanthophyllomyces as a valuable industrial production platform (Schmidt et al. 2011; Verdoes et al. 2003).

The genome of Xanthophyllomyces has been fully sequenced and recently published (Sharma et al. 2015). The main biosynthetic pathways derived from acetyl-CoA are terpenoids and lipids. The genes involved in ergosterol (the main sterol), (Loto et al. 2012), astaxanthin (Alcaíno et al. 2008; Ojima et al. 2006; Verdoes et al. 1999) and fatty acids have been identified (Sharma et al. 2015). The biosynthetic genes assigned providing valuable tools for the metabolic engineering of the pathway. Carotenoids are forty-carbon $\left(\mathrm{C}_{40}\right)$ terpenoids formed from the $\mathrm{C}_{5}$ precursor isopentenyl diphosphate (IPP), making them members of the isoprenoid family of natural products. In Xanthophyllomyces, terpenoids are synthetize via the mevalonate pathway (Sharma et al. 2015). The IPP derived prenyl lipids are formed by the action of farnesyl diphosphate synthase (FPPS), the farnesyl diphosphate (FPP) formed which has a $\mathrm{C}_{15}$ alkane chain is the precursor for sterols. Further extension of prenyl chain to the $\mathrm{C}_{20}$ geranylgeranyl diphosphate (GGPP) which is formed by GGPP synthase (GGPPS) occurs in Xanthophyllomyces. Condensation of GGPP catalysed by phytoene synthase results in the formation of phytoene, a colourless $\mathrm{C}_{40}$ carotene. Four desaturation and two cyclization catalysed by phytoene desaturase and lycopene cyclase, respectively, form $\beta$-carotene. Astaxanthin synthase a P450-type mixed function oxygenase produces two hydroxylations and two ketolations at the $3,3^{\prime}$ and $4,4^{\prime}$ positions on the $\beta$-ionone ring, respectively to form astaxanthin. Carotenoids are produced within the organism to provide protection against photooxidative stress and inactivated free radicals via electron transfer (Niyogi et al. 1997). It is the formation of isoprenoids in general and particularly those beyond GGPP that give Xanthophyllomyces the unprecedented opportunity to be the platform of choice for the production of high value isoprenoids that can be utilised across multiple industrial sectors.

Metabolomics/metabolite profiling is a key component of the omics toolkit; enabling the large scale determination of steady state levels of metabolites as well as the perturbations in these metabolites resulting from changes in their environment or in response to pathway manipulation (Villas-Bôas et al. 2005). Key attributes of microbial metabolomics systems include the effective arrest (quenching) of metabolism, efficient extraction of metabolites across a broad dynamic range and the analysis/visualisation of the data (Mashego et al. 2007). These metabolite profiling techniques have been developed in industrial/model microorganisms like Escherichia coli (Prasad Maharjan and Ferenci 2003; Taymaz-Nikerel et al. 2009; Winder et al. 2008) and Saccharomyces cerevisiae (Villas-Bôas et al. 2005). However, Xanthophyllomyces has the unique characteristics of being a naturally high terpenoid producer and a "Crabtree negative" industrial yeast (Van Urk et al. 1989). can In the present study a metabolite profiling pipeline for Xanthophyllomyces has been established and its utility demonstrated. With these procedures in place biochemical characterisation of strain improvement outputs and associated Genome Scale Metabolic Reconstruction (GSMR) networks can now be performed

\section{Results}

\subsection{Analysis of quenching methods}

Both primary or intermediary metabolism have an extremely fast turnover (Winder et al. 2008). Efficient quenching methods are essential for accuracy when determining steady state metabolite levels in microorganisms. Previous studies addressing the quenching of metabolism in yeasts (Canelas et al. 2008), have focused on four different quenching methods to evaluated the arrest of metabolism in cultured cells. The comparator (control material) being cellular material harvested by centrifugation without the application of the quenching methods prior to freezing the biomass has been described in the methods section.

All quenching methods showed a recovery value between 0.77 and 1.22 , with the arrest of metabolism in Xanthophyllomyces, based on the recovery of the authentic standards used (Fig. 1). The effect of the quenching methods over the general metabolism was also evaluated. The complete overview of the polar extraction of metabolites was provided from the GC-MS analysis, which through library matching showed a high number of identified compounds in samples treated with the wash $\mathrm{NaCl}$ solution (W). In this case the initial searches identified over 90 


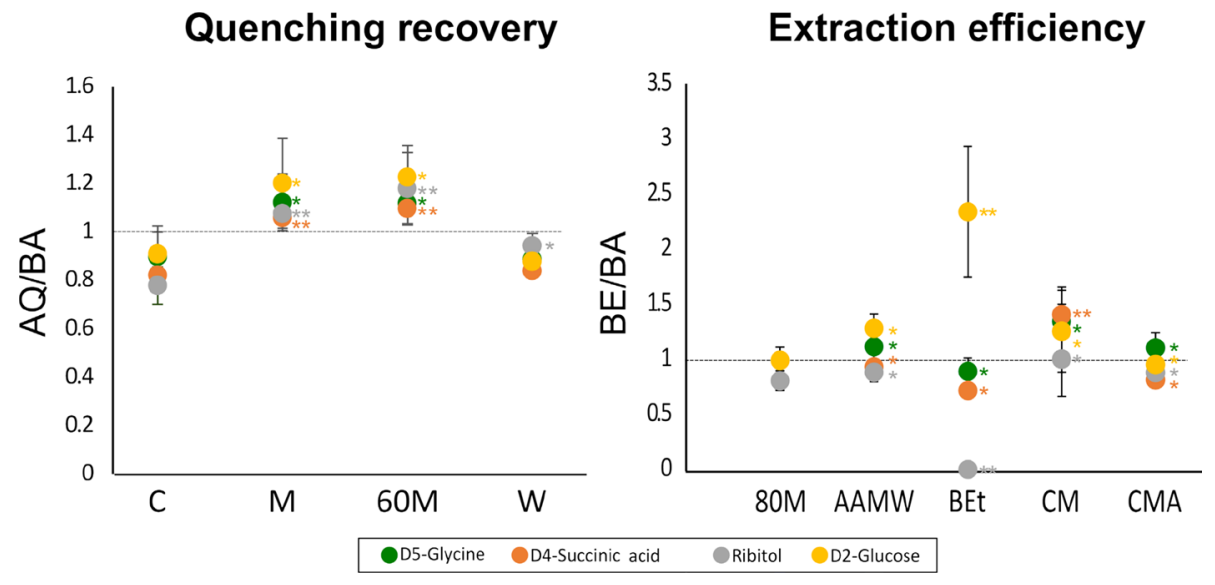

Fig. 1 Quenching recovery and extraction efficiency calculated as the ratio of the standards spiked after quenching (AQ) or before extraction (BE) and before analysis (BA) respectively. $C$ control, $60 M$ cold $60 \%$ methanol in $0.9 \% \mathrm{NaCl}, M$ cold methanol, $W$ washing with $0.9 \% \mathrm{NaCl}, 80 M 80 \%$ methanol, $A A M W$ acidified acetonitrile:methanol:water, Bet Boiling ethanol, $C M$ chloroform:methanol, $C M A$ chloroform:methanol acidified. *p $\backslash 0.05$; **p $\backslash 0.01$ [C method used as control for recovery and $80 \mathrm{M}$ method used as control for efficiency]

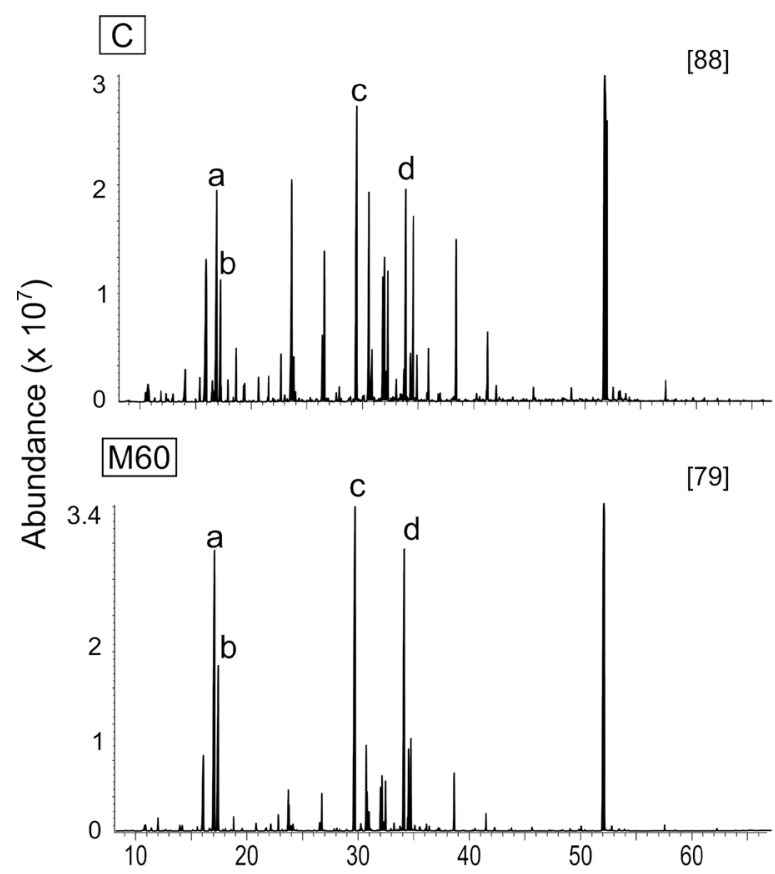

Fig. 2 A comparison of the GC-MS profiling following extracts prepared with different quenching methods. In squares: $C$ control, $60 M$ cold $60 \%$ methanol in $0.9 \% \mathrm{NaCl}, M$ cold methanol, $W$ washing with

targeted polar compounds (Fig. 2). This quenching method provided the most satisfactory results (in terms of compound numbers) and was logistically appropriate for the study of Xanthophyllomyces and its industrial exploitation.
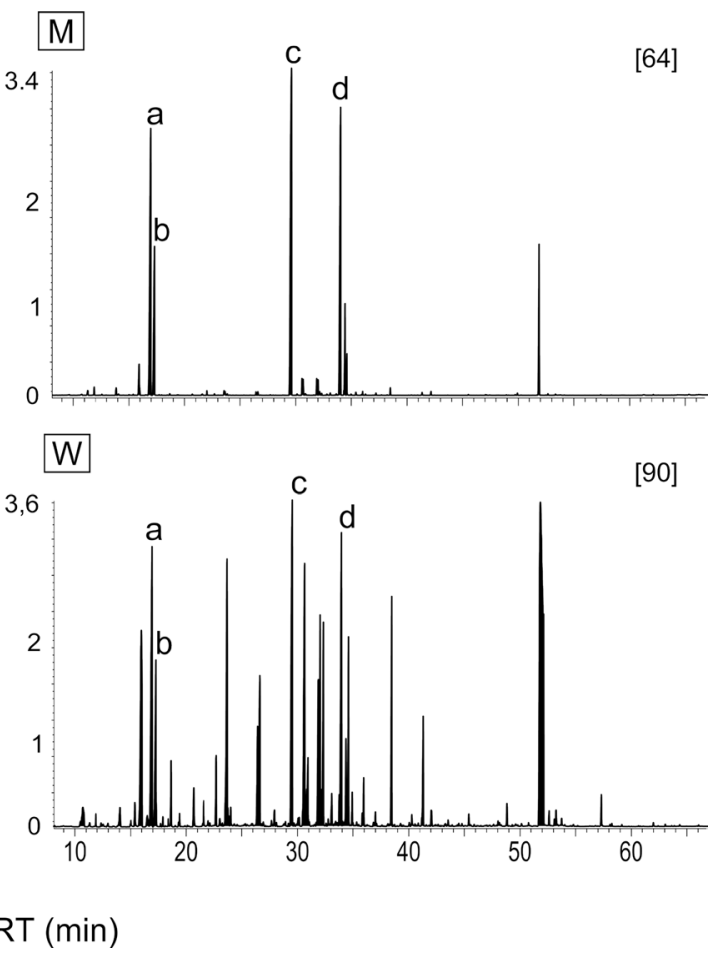

$0.9 \% \mathrm{NaCl}$. In brackets: number of targeted compound in the library. Standards added indicated by letters: $\mathbf{a} \mathrm{D}_{5}$-glycine; $\mathbf{b} \mathrm{D}_{4}$-succinic acid; c ribitol; $\mathbf{d} \mathrm{D}_{2}$-glucose

\subsection{Extraction optimisation}

The $80 \%$ methanol $(80 \mathrm{M})$, acidified acetonitrile:methanol:water (AAMW), chloroform:methanol (CM) and chloroform:methanol acidified (CMA) methods all showed a good performance. 
The highest extraction efficiency values for all the standards used were achieved with the CM method (Fig. 1). The boiling ethanol (Bet) method gave the greatest differences in efficiency. However it was the most effective method for $\mathrm{D}_{2}$-glucose extraction but the important standard ribitol could not be detected.

A comparison between all the metabolites extracted by the extraction methods tested showed important differential extraction properties between the compound classes (Fig. 3). The chloroform/methanol-based methods (CM and CMA), were more efficient at extracting polar compounds, such as amino acids, organic acids, sugars and sugar derivatives, and free fatty acids. The highest efficiency for glycerolipids and unknown non-polar compounds was detected using the 80M and AAMW approaches. BEt showed less efficiency for all the classes of metabolites detected/tested.

Base on extraction efficiency, logistically properties and the range of metabolites detected the CM method in combination with the $\mathrm{NaCl}$ wash step was the most convenient procedure for extraction in Xanthophyllomyces.

\subsection{GC-MS library for xanthophyllomyces dendrorhous}

The GC-MS library for Xanthophyllomyces was created in an AMDIS format, using retention time locking and concurrent chromatography with a hydrocarbon standard mixture, in order to generate of retention indices (Table S1). Identification of chromatographic components was carried out by firstly performing automated matching of mass spectrum to those present in the NIST and/or Golm metabolome databases (http://gmd.mpimp-golm.mpg.de/analysisinput.aspx). These putative matches were then confirmed with authentic standards. Alternatively, authentic standards representing metabolites known to be present in Xanthophyllomyces and possessing chemistry amenable to GC-MS analysis, were chromatographed and their characteristic on-line MS spectrum added to the library. The library presently contains more than 400 metabolites and its curation is on-going. This analytical platform allowed the identification and quantification of 76 metabolites (Table S2) among the polar and non-polar extracts of the different ages Xanthophyllomyces cultures. In accordance with the recommendations proposed

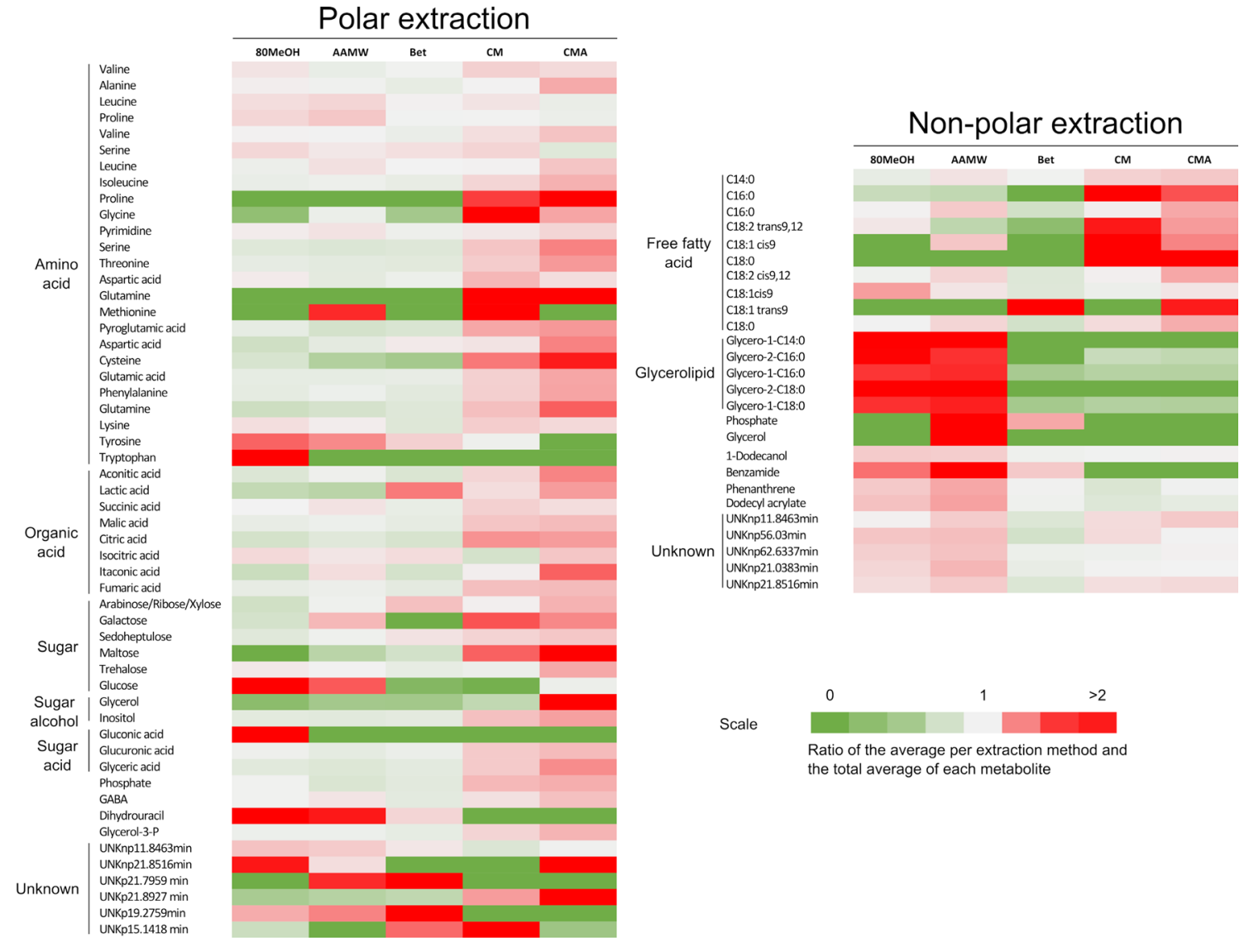

Fig. 3 Heat map illustrating the levels of polar and non-polar metabolites obtained with different extraction methods. Values were normalized to the dry weight of samples and pareto-scaled. Values represent means and standard deviations obtained from six replicates 
by the metabolomics standards initiative (Bino et al. 2004; Sumner et al. 2007), chromatographic components were annotated, providing valuable metadata and validation of peak identities (Table S1). To provide an indication of the biological and technical variation within the system a series of analyses were carried out on material cultivated under standardised conditions.

Over a 100 GC-MS peaks were detected in the extracts of the quenching samples in Xanthophyllomyces strains, 68 were identified as known components and 8 unknown. Amino acids, fatty acids, organic acids and sugars are mainly represented. Furthermore, the terpenoids, $\beta$-carotene, phytoene, astaxanthin, ubiquinone (Q9) and ergosterol, were detected by UPLC-PDA. The metabolites identified included a range of intermediates and end products of the central and secondary metabolism (Table 1). This enabled the construction of a quantitative biochemical network for Xanthophyllomyces for the display of changes in steady state levels of detectable metabolites present.

\subsection{Changes in steady state metabolites during the production of Xanthophyllomyces biomass}

In this study selected time points across the characteristic growth profile of Xanthophyllomyces have been used representing the lag ( 1 day) and exponential phases (2 days) as well as early (4 days) and late stationary (7 days) phases (Fig. S1). Visual inspection of the GS-MS chromatograms indicated similar profiles for all cultures (Fig. S2). A data matrix was created combining the variables from all the analytical platforms used and then the data subjected to PCA, in order to identify variables (metabolites) contributing to the overall changes in chemical composition between the different-growth stages (Fig. 4). The score plot from the PCA separate the cultures into three groups, 1,2 and 4-7 days of cultivation. These data indicated that despite similar visual chromatographic profiles that the comparative chemical composition among all the cultures is significantly different. The loading plot of the PCA data indicated that the separation of the cultures could be attributed to several metabolites or sectors of metabolism. For example, glycerolipids, amino acid metabolites, sugars and some unknown compounds contributed to the clustering of the 1 day old culture from the other stages. While mainly free fatty acids, glycerolipids, ubiquinone, maltose, lysine and GABA contributed to the clustering of the 2 days old culture. The clustering of the 4 and 7 day old cultures was due to the carotenoids and members of fatty acid, organic acid, amino acid and unknown families.

More detailed analysis of the individual changes in metabolites was achieved through pair-wise statistical analysis, using the significance derived from Student's $t$ tests
(Table 1). Glycerophospholipids, sugars, polyols, sugar alcohols, terpenoids and phosphorylated compounds decrease significantly with age of culture. Fatty acids increased in their quantity during the exponential phase and decreased during the stationary phase. While organic acids, sugar acids and non-amino acid $\mathrm{N}$-containing compounds increase significantly 2.5 -fold, threefold and fourfold, respectively. Terpenoids decreased in their total amount at day 2 and 4 of cultivation followed to a measurable increment from day 4 to day 7 of culture.

More extensive analysis of the terpenoids (Fig. 5) indicated the strongly influence of ergosterol over the total amount of terpenoids produced. Ubiquinone exhibited lower amounts at the early stationary phase and reach the same level of lag and exponential phase at 7 days of culture. Astaxanthin is the main carotenoid in Xanthophyllomyces (Johnson 2003; Miller et al. 1976), phytoene and $\beta$-carotene are precursors which are identified in the method described. The amount of phytoene decreases over cultivation in comparison to day 1 of cultivation. $\beta$-Carotene is present and consistent at a similar levels throughout growth. Astaxanthin increased constantly over the 7 days of culture, constituting a 3.5-fold increase in the amount compared to day 1 of cultivation.

These changes were then visualised by representing graphically the changes of metabolites and correlating with the growth curve of the cultures in a heat map (Fig. 6). The clustering of the samples distinguish two main metabolite groups based on the behaviour along the culture growth. One group correspond to those metabolites accumulated during the stationary phase: TCA, "short-chain" (14-17 carbon chain) saturated fatty acids, astaxanthin, $\beta$-carotene and most of the amino acids clustered in this group. The second cluster grouped metabolites synthetize rapidly during lag and exponential phase and metabolised during the stationary phase: "Long-chain" (more than 18 carbon chain) saturated fatty acids, glycerolipids, some amino acids, ergosterol and phytoene.

\subsection{Metabolic correlations associated with astaxanthin production}

Correlation analysis using Pearson coefficients was carried out and displayed in a heat map format (Fig. 7) associated analysis of compound patterns was made to correlate astaxanthin, with the rest of the metabolites (Table S3). Sugars, glycerolipids, "long-chain" saturated fatty acids, phytoene and ergosterol diminish in quantity as astaxanthin formation was favoured. Unsaturated and "long-chain" fatty acids, ubiquinone, some sugars and some amino acids did not show a significant positive or negative correlation with astaxanthin biosynthesis. The predominant metabolites/chemical classes observed over the cultivation period are summarised in Fig. 
Table 1 Relative metabolite changes occurring at designated growth stages (exponential and early and late stationary stages) compared with a standardised 1 day (lag stage) culture as control

\begin{tabular}{|c|c|c|c|c|c|c|c|}
\hline \multirow[t]{2}{*}{ Metabolite } & \multicolumn{3}{|l|}{ Fold change } & \multirow[t]{2}{*}{ Metabolite } & \multicolumn{3}{|l|}{ Fold change } \\
\hline & 2 Days & 4 Days & 7 Days & & 2 Days & 4 Days & 7 Days \\
\hline Amino acids & & & & Phosphate & & & \\
\hline Alanine & $0.48 \pm 0.28$ & $0.69 \pm 0.35$ & $0.29 \pm 0.17$ & Phosphate & $0.40 \pm 0.09$ & $0.61 \pm 0.14$ & $0.74 \pm 0.27$ \\
\hline Aspartic acid & $0.70 \pm 0.13$ & $1.00 \pm 0.19$ & $1.16 \pm 0.18$ & Polyols & & & \\
\hline Beta-alanine & $1.17 \pm 0.48$ & $14.36 \pm 4.35$ & $1.19 \pm 0.35$ & 1-Dodecanol & $1.38 \pm 0.36$ & $0.99 \pm 0.14$ & $1.02 \pm 0.12$ \\
\hline Cysteine & $0.84 \pm 0.12$ & $2.94 \pm 0.26$ & $6.57 \pm 2.34$ & Ribitol & $0.79 \pm 0.11$ & $0.15 \pm 30.01$ & A \\
\hline GABA & $1.48 \pm 0.28$ & $0.20 \pm 0.11$ & $0.20 \pm 0.05$ & Total & $0.84 \pm 0.08$ & $0.22 \pm 0.01$ & $0.09 \pm 0.01$ \\
\hline Glutamic acid & $0.84 \pm 0.11$ & $0.76 \pm 0.20$ & $0.81 \pm 0.14$ & Phosphorylated compounds & & & \\
\hline Glutamine & $*$ & $\mathrm{P}$ & $\mathrm{P}$ & Glucose-6-phosphate & $0.78 \pm 0.22$ & $0.25 \pm 0.02$ & $0.28 \pm 0.12$ \\
\hline Glycine & $0.48 \pm 0.03$ & $1.81 \pm 0.16$ & $0.83 \pm 0.49$ & Glycerol-3-phosphate & $0.55 \pm 90.04$ & $0.57 \pm 90.02$ & $0.49 \pm 0.08$ \\
\hline Homoserine & A & $0.96 \pm 0.27$ & $0.54 \pm 0.26$ & Total & $0.56 \pm 0.04$ & $0.56 \pm 0.02$ & $0.48 \pm 0.08$ \\
\hline Isoleucine & $\mathrm{P}$ & $\mathrm{P}$ & $\mathrm{P}$ & Sugars & & & \\
\hline Leucine & $0.74 \pm 0.06$ & $1.39 \pm 10.15$ & $1.39 \pm 0.20$ & Arabinose/ribose/xylose & $0.61 \pm 10.08$ & $0.61 \pm 10.04$ & $0.19 \pm 0.11$ \\
\hline Lysine & $1.18 \pm 0.03$ & $0.59 \pm 0.09$ & $1.07 \pm 0.53$ & Disaccharide $56.509 \mathrm{~min}$ & $0.94 \pm 0.15$ & $0.39 \pm 0.04$ & $0.29 \pm 0.06$ \\
\hline Methionine & $0.48 \pm 0.09$ & $1.67 \pm 0.37$ & $0.97 \pm 0.45$ & Disaccharide $56.902 \mathrm{~min}$ & A & A & A \\
\hline Phenylalanine & $0.83 \pm 0.12$ & $3.51 \pm 0.66$ & $2.00 \pm 0.61$ & Fructose & $0.55 \pm 0.04$ & A & A \\
\hline Proline & $0.63 \pm 0.09$ & $0.24 \pm 0.06$ & $0.59 \pm 0.05$ & Glucose & $0.30 \pm 0.06$ & $0.07 \pm 0.01$ & $0.06 \pm 0.01$ \\
\hline Putrescine & A & A & A & Maltose & $\mathrm{P}$ & $*$ & $*$ \\
\hline Pyroglutamic acid & $0.88 \pm 0.14$ & $0.86 \pm 0.40$ & $0.87 \pm 0.33$ & Me-fructose & A & A & A \\
\hline Serine & $0.89 \pm 0.16$ & $2.39 \pm 0.26$ & $2.52 \pm 0.78$ & Me-galactose & $\mathrm{P}$ & $\mathrm{P}$ & $\mathrm{P}$ \\
\hline Threonine & $0.36 \pm 0.04$ & $0.37 \pm 0.10$ & $0.27 \pm 0.12$ & Sedoheptulose & $*$ & $*$ & $\mathrm{P}$ \\
\hline Valine & $0.79 \pm 0.26$ & $0.94 \pm 0.15$ & $1.32 \pm 0.18$ & Trehalose & $0.68 \pm 0.17$ & $0.67 \pm 0.15$ & $0.75 \pm 0.11$ \\
\hline Total & $0.71 \pm 0.09$ & $1.05 \pm 0.05$ & $0.88 \pm 0.15$ & Total & $0.59 \pm 10.13$ & $0.52 \pm 0.11$ & $0.57 \pm 0.08$ \\
\hline Fatty acids & & & & Sugar acids & & & \\
\hline $\mathrm{C} 14: 0$ & $\mathrm{P}$ & $\mathrm{P}$ & $\mathrm{P}$ & Erythronic acid & A & A & A \\
\hline C15:0 & $1.11 \pm 0.15$ & $1.84 \pm 0.30$ & $1.96 \pm 0.19$ & Glucuronic acid & $*$ & $\mathrm{P}$ & $\mathrm{P}$ \\
\hline C16:0 & $1.37 \pm 0.23$ & $0.97 \pm 0.05$ & $0.91 \pm 0.13$ & Glyceric acid & $1.38 \pm 0.31$ & $2.98 \pm 0.57$ & $1.63 \pm 0.28$ \\
\hline $\mathrm{C} 17: 0$ & $1.34 \pm 0.46$ & $2.81 \pm 1.72$ & $2.16 \pm 0.82$ & Total & $0.82 \pm 0.19$ & $2.99 \pm 0.49$ & $2.05 \pm 0.28$ \\
\hline C18:0 & $1.27 \pm 0.16$ & $0.63 \pm 0.02$ & $0.57 \pm 0.14$ & Sugar alcohols & & & \\
\hline C18:1 trans9 & $1.74 \pm 0.36$ & $1.43 \pm 0.27$ & $1.25 \pm 0.26$ & Glycerol & $0.59 \pm 0.06$ & $0.00 \pm 0.00$ & $0.01 \pm 0.00$ \\
\hline C18:1cis9 & $1.41 \pm 0.35$ & $0.95 \pm 0.07$ & $0.99 \pm 0.19$ & Inositol & $0.71 \pm 0.01$ & $2.01 \pm 0.09$ & $1.61 \pm 0.43$ \\
\hline $\mathrm{C} 18: 2$ cis 9,12 & $1.30 \pm 0.20$ & $1.12 \pm 0.08$ & $1.02 \pm 0.12$ & Total & $0.62 \pm 10.05$ & $0.45 \pm 0.02$ & $0.37 \pm 0.10$ \\
\hline $\mathrm{C} 18: 2 \operatorname{trans} 9,12$ & $2.72 \pm 0.72$ & $2.04 \pm 1.01$ & $1.69 \pm 1.28$ & Non-amino acid N-containi & ing compounds & & \\
\hline C20:0 & $1.43 \pm 0.19$ & $0.84 \pm 0.05$ & $0.70 \pm 0.15$ & Dihydrouracil & $*$ & $\mathrm{P}$ & $\mathrm{P}$ \\
\hline $\mathrm{C} 22: 0$ & $1.68 \pm 0.26$ & $1.28 \pm 0.21$ & $1.26 \pm 0.39$ & Pyrimidine & $1.24 \pm 0.09$ & $2.97 \pm 10.39$ & $2.55 \pm 0.60$ \\
\hline $\mathrm{C} 24: 0$ & $2.09 \pm 0.39$ & $1.53 \pm 0.21$ & $1.41 \pm 0.33$ & Total & $1.24 \pm 0.09$ & $4.14 \pm 0.54$ & 3.9790 .73 \\
\hline Total & $1.41 \pm 0.24$ & $1.11 \pm 0.08$ & $1.03 \pm 0.16$ & Terpenoids & & & \\
\hline Glycerophospholipids & & & & Astaxanthin & $1.63 \pm 10.28$ & $2.30 \pm 0.32$ & $3.32 \pm 0.30$ \\
\hline Glycero-1-C14:0 & $0.95 \pm 0.15$ & $0.75 \pm 0.16$ & $0.60 \pm 0.09$ & Beta-carotene & $1.09 \pm 30.20$ & $1.02 \pm 0.22$ & $1.10 \pm 0.15$ \\
\hline Glycero-1-C15:0 & $0.95 \pm 0.15$ & $0.79 \pm 0.07$ & $0.80 \pm 0.07$ & Ergosterol & $0.73 \pm 30.15$ & $0.44 \pm 0.06$ & $0.60 \pm 0.09$ \\
\hline Glycero-1-C18:0 & $0.93 \pm 0.16$ & $0.75 \pm 0.06$ & $0.79 \pm 0.08$ & Phytoene & $0.49 \pm 0.13$ & $0.49 \pm 10.24$ & $0.12 \pm 0.13$ \\
\hline Glycero-1-C18:1cis9 & $0.85 \pm 0.28$ & $0.66 \pm 0.18$ & $0.45 \pm 0.12$ & Ubiquinone & $1.03 \pm 10.20$ & $0.49 \pm 0.10$ & $0.87 \pm 0.12$ \\
\hline Glycero-2-C16:0 & $0.84 \pm 0.18$ & $0.85 \pm 0.11$ & $0.89 \pm 90.06$ & Total carotenoids & $0.98 \pm 0.15$ & $1.03 \pm 0.14$ & $1.45 \pm 0.12$ \\
\hline Glycero-2-C18:0 & $0.81 \pm 0.13$ & $0.87 \pm 0.09$ & $0.90 \pm 0.07$ & Total terpenoids & $0.76 \pm 0.15$ & $0.48 \pm 0.07$ & $0.67 \pm 0.09$ \\
\hline Total & $0.94 \pm 0.15$ & $0.77 \pm 0.07$ & $0.80 \pm 0.07$ & Unknown compounds & & & \\
\hline Organic acids & & & & UNKnp11.8463min & $1.03 \pm 0.20$ & $2.84 \pm 2.67$ & $0.31 \pm 0.34$ \\
\hline Aconitic acid & $1.06 \pm 0.43$ & $5.17 \pm 1.26$ & $7.45 \pm 3.10$ & UNKnp21.0383min & $1.13 \pm 0.11$ & $2.23 \pm 0.97$ & $0.50 \pm 0.18$ \\
\hline Citric acid & $0.84 \pm 0.21$ & $2.07 \pm 0.31$ & $3.50 \pm 1.14$ & UNKnp56.03min & $1.01 \pm 0.17$ & $0.88 \pm - \pm 0.06$ & $0.86 \pm 0.12$ \\
\hline D-Arabinonic acid & A & $\mathrm{A}$ & A & UNKnp62.6337min & $1.20 \pm 0.18$ & $1.13 \pm 0.16$ & $0.95 \pm 0.13$ \\
\hline
\end{tabular}


Table 1 (continued)

\begin{tabular}{|c|c|c|c|c|c|c|c|}
\hline \multirow[t]{2}{*}{ Metabolite } & \multicolumn{3}{|l|}{ Fold change } & \multirow[t]{2}{*}{ Metabolite } & \multicolumn{3}{|l|}{ Fold change } \\
\hline & 2 Days & 4 Days & 7 Days & & 2 Days & 4 Days & 7 Days \\
\hline Fumaric acid & $2.15 \pm 0.14$ & $3.21 \pm 0.55$ & $2.57 \pm 0.45$ & UNKp15.1418min & $0.33 \pm 0.07$ & $0.13 \pm 0.04$ & $2.21 \pm 1.38$ \\
\hline Itaconic acid & $*$ & $\mathrm{P}$ & $\mathrm{P}$ & UNKp21.7959min & A & A & $0.52 \pm 40.12$ \\
\hline Lactic acid & $0.46 \pm 0.36$ & A & $\mathrm{A}$ & UNKp21.8927min & $*$ & $\mathrm{P}$ & $\mathrm{P}$ \\
\hline Malic acid & $1.33 \pm 0.05$ & $2.05 \pm 0.25$ & $2.00 \pm 0.34$ & UNKp27.5420min & A & $1.40 \pm 0.45$ & A \\
\hline Succinic acid & $0.74 \pm 0.07$ & $0.60 \pm 0.10$ & $0.64 \pm 0.08$ & Total & $0.82 \pm 0.11$ & $2.42 \pm 0.85$ & $1.91 \pm 0.25$ \\
\hline Total & $1.00 \pm 0.11$ & $1.78 \pm 0.15$ & $2.38 \pm 0.54$ & & & & \\
\hline
\end{tabular}

Data were compiled from multiple analytical platforms. The ratio data are presented as mean \pm SD. Student's $t$ test analysis was carried out. Significant changes are presented in bold $(\mathrm{P}$ value $<0.05)$. $\mathrm{P}$, when a metabolite is present in the sample and not in the control at the concentration used; A, theoretical value when a metabolite is unique to control at the concentration used; *, indicates metabolite no detected in both cultures at the concentration used
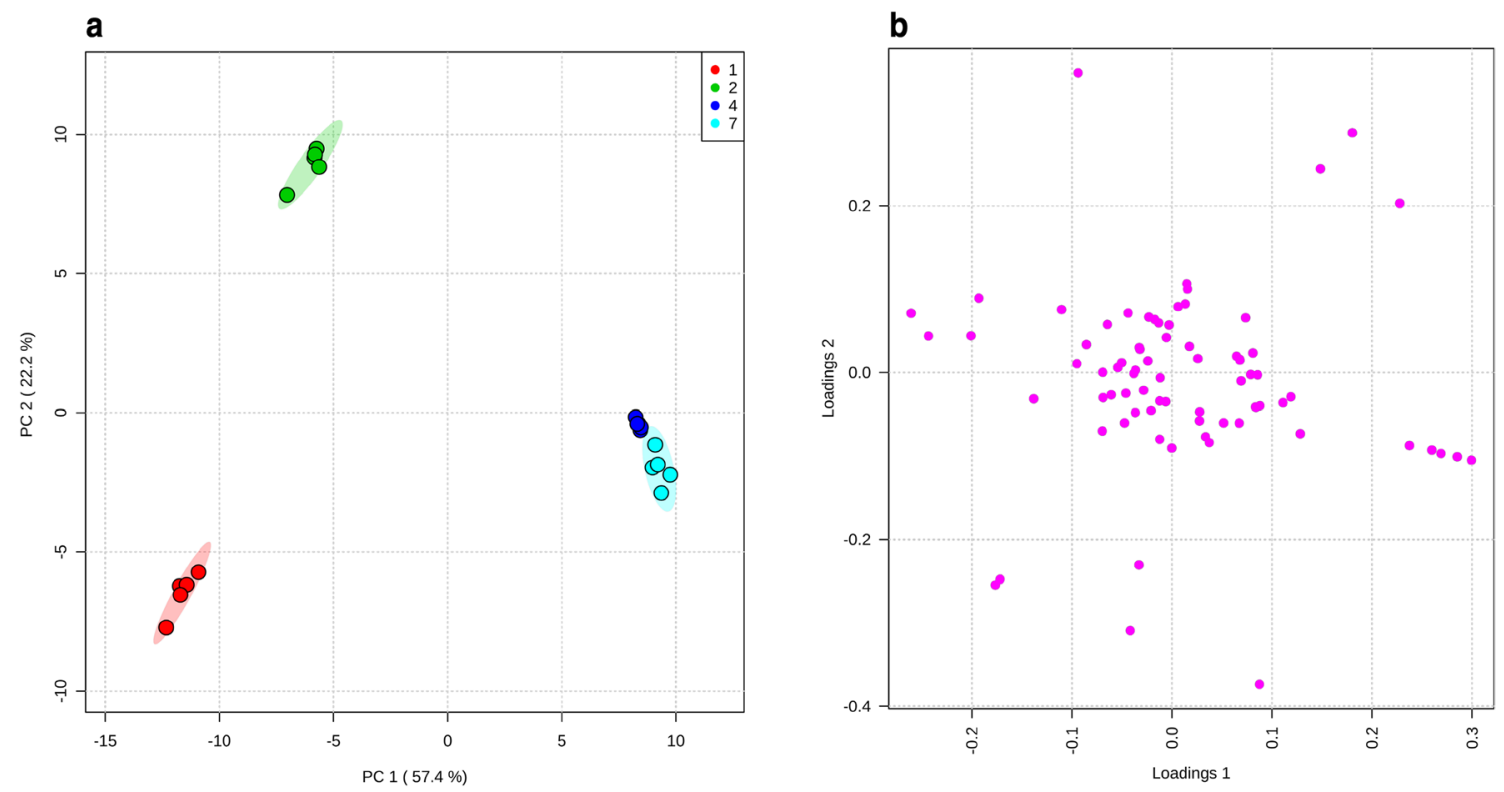

Fig. 4 Principal component analysis. a Score plot showing the clustering pattern of Xanthophyllomyces wild-type strain at 1 (blue), 2 (yellow), 4 (green) and 7 (red) days of culture; b loading plot showing those metabolites responsible for the clustering observed in Xanthophyllomyces

S3. These data clearly displayed the predominant of metabolites present during the stationary phase.

\section{Discussion}

In the present study a metabolite profiling workflow has been reported for the non-conventional industrial yeast Xanthophyllomyces which naturally produces astaxanthin, a high value carotenoid. Of the quenching methods tested washing with $\mathrm{NaCl}$ solution (W), followed by chloroform:methanol (CM) extraction were the most reliable method to arrest metabolites in Xanthophyllomyces, based on quenching recovery (0.84-0.94), extraction efficiency (1.00-1.41), number of targeted compounds (90) and chemical families representation. The need for specific quenching procedures for the microorganisms in question has been reported by (Mashego et al. 2007) and the present study corroborates these findings. Different methodology has been reported for metabolite profiling analysis in Xanthophyllomyces (Martinez-Moya et al. 2015). Pre-chilled methanol (60\% $\mathrm{v} / \mathrm{v}$ ) for quenching the metabolism has been used previously (Martinez-Moya et al. 2015), similar to the quenching methods used in this study. In comparison to the evaluation of methods used for bacteria (Perez-Fons et al. 2014) all the quenching methods tested in the present study showed 


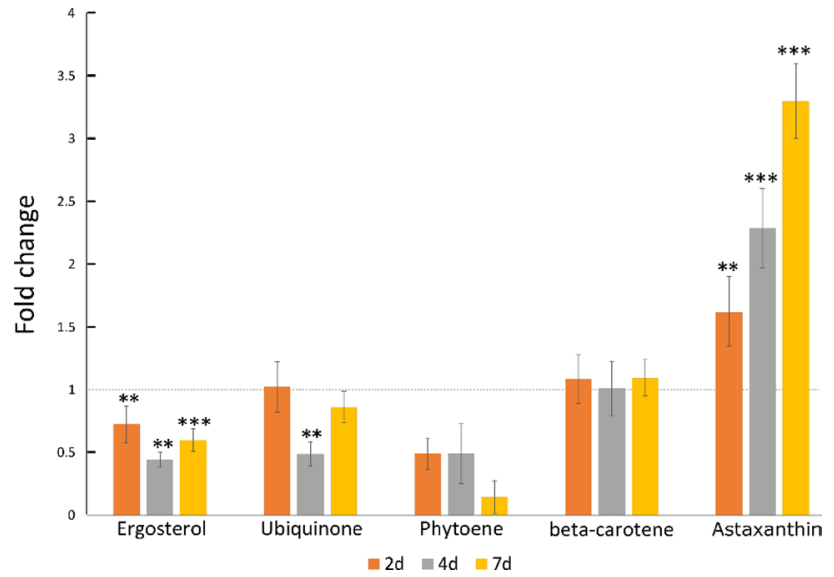

Fig. 5 Specific changes in detectable terpenoids during growth. Data obtained by UPLC-PDA analysis. Fold changes of terpenoids using 1 day culture as control. *p\0.05;**p $\backslash 0.01 ; * * * p \backslash 0.001$

a very similar recovery parameters for the standards, suggesting that the metabolism of the yeast cell does not have the same rapidity and less susceptible to conditions adopted during harvesting. The quenching methods using methanol ( $\mathrm{M}$ and $\mathrm{M60}$ ), typically result in reduced metabolites, presumably because the approach, although rapid, can actually leach metabolites from the cell. Therefore, the simple washing of the cell with $0.9 \%(\mathrm{w} / \mathrm{v}) \mathrm{NaCl}(\mathrm{W})$ to remove media components gave more representative results due to more efficient quenching of Xanthophyllomyces without perturbing metabolite levels.

It is important that the extraction method used provides a comprehensive representation of the main metabolite classes. Boiling ethanol was reported as an efficient extraction method in Saccharomyces cerevisiae (Gonzalez et al. 1997). In our study this method showed greatest variability in efficiency. This extraction method was also the least effective for Mycobacteria and Bacillus respectively (Drapal et al. 2014; Perez-Fons et al. 2014). Previous extraction methods applied to Xanthophyllomyces (Martinez-Moya et al. 2015) or to Haematococcus pluvialis (Lv et al. 2016) are based on sequential methanol:water extractions and focussed on polar metabolites. The implications associated with fatty acid components, glycerophospholipids, carotenoids and sterols in membranes has been reported (Gruszecki and Strzałka 2005), the presence of these compounds in the non-polar extraction validates the use of the present extraction method for non-polar components. Of the five different tested extraction methods showed altered affinities for different classes of metabolites. In Xanthophyllomyces, the dominating fatty acids are palmitic, oleic and linoleic acid (C16:0, C18:1, cis-9 and C18:2, cis-9,12, respectively) (Sharma et al. 2015), those compounds were clearly represented after CM extraction method. The efficiency of methanol:chloroform based method for the extraction of polar and non-polar metabolites has already been documented (Winder et al. 2008).

The construction of the AMDIS library is a valuable resource, enabling the identification of almost 70 metabolites from the chromatographic components. The optimised CM extraction method combined with UPLC, for terpenoids mainly, and GC-MS, for primary/intermediary metabolites, is a robust platform for studying the metabolism of Xanthophyllomyces and can hopefully be extended to other nonconventional industrially useful fungi.

In order to test the potential of the optimised system and define a baseline of steady state metabolite levels synthesised by Xanthophyllomyces under standardised laboratory cultivation conditions, the optimised CM method was used to analyse the biomass generated over the different growth phases (lag, exponential and early and late stationary phases) of the Xanthophyllomyces growth cycle.

From the collective PCA plots specific metabolite changes could be associated with different stages of growth. Direct pairwise comparisons of metabolites indicated the main metabolite changes contributing to the clustering were glycerophospholipids, sugars, polyols, sugar alcohols, terpenoids and phosphorylated compounds which decrease significantly with the time. Fatty acids increase in quantity during the exponential phase and decreased during the stationary phase. While organic acids, sugar acids and nonamino acid $\mathrm{N}$-containing compounds increase significantly. Metabolite global changes were also reported previously in Xanthophyllomyces at different growth stages (MartinezMoya et al. 2015).

Carotenoids are produced within the organism to provide protection against photooxidative stress and inactivated free radicals via electron transfer (Niyogi et al. 1997). The initiation of oxidative stress also stimulates the synthesis of carotenoids in fungi (Gessler et al. 2007). In Xanthophyllomyces, during late exponential phase carotenogenic-related and redox proteins have been reported to be more abundant than in previous growth stages (Martinez-Moya et al. 2015). Correlation analysis of metabolites to the astaxanthin (Fig. 7 and Table S3) lead to the separation of metabolites into two groups. Astaxanthin correlated positively with intermediary metabolism (TCA and amino acids). The accumulation of the TCA intermediates during the stationary phase may indicate a lower activity than in previous phases of cultivation. TCA and high respiratory cycles are related with ROS production, which increase the astaxanthin biosynthesis in Xanthophyllomyces (Schroeder and Johnson 1993). The close relation of carotenoid production, the TCA cycle and stress response has been reported with differential protein expression between wild-type and mutant strains (Barbachano-Torres et al. 2014).

In Saccharomyces cerevisiae proline protects the cell against different stress conditions such as oxidative or light 


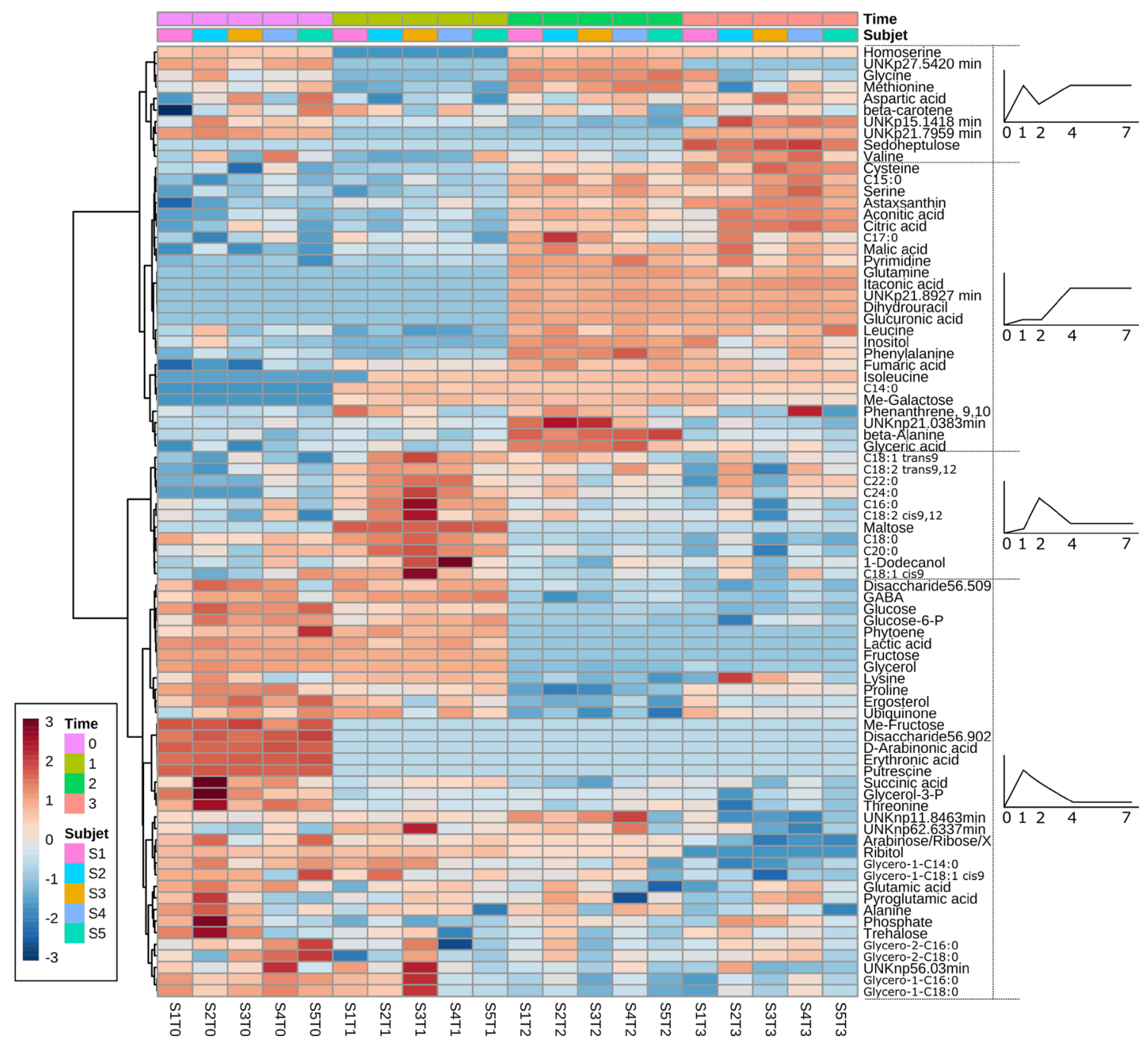

Fig. 6 Heat map of the metabolite changes throughout the growth of Xanthophyllomyces in laboratory shake flask cultivation. Dashed line separate groups of metabolites with different trend (right) along of the culture; graphs represent the trend of the metabolites along the

induced stresses (Takagi 2008). In Xanthophyllomyces, proline diminished with the time of the culture (Table 1) correlating negatively with astaxanthin formation (Fig. 7 and Table S3), astaxanthin could provide more effective protection reducing the need for proline. The same effect has been described in the astaxanthin producing algae Haematococcus pluvialis (Lv et al. 2016).

Similar metabolite profiling techniques have been applied to a distant filamentous fungus Phycomyces blakesleeanus revealing global changes across intermediary metabolism of the mutants (Alcalde and Fraser 2016). Classical mutagenesis and genetic engineering have produced a plethora of strains with altered or enhanced carotenoid content in Xanthophyllomyces (Schmidt et al. 2011). A robust metabolite profiling method will enable a better understanding of the changes in steady state levels in Xanthophyllomyces. culture; numbers under the graph represent the day of the culture. The heat map was created using the server MetaboAnalyst and presented in Inkscape

A microbial-derived production platform for terpenoids could potentially replace plant-derived and chemically synthesised industrial methods (Ma et al. 2016). Nevertheless, the steady-state kinetic formation of terpenoids in microbial systems is still poorly understood (Zhu et al. 2014). Understanding these holistic changes will enable us to decipher the correlated metabolite pathways that exist and facilitate the design of new strategies for the exploitation of Xanthophyllomyces as a cell factory for high value isoprenoids. Moreover, important advances in proteomic (Martinez-Moya et al. 2011, 2015) and genomic (Bellora et al. 2016; Sharma et al. 2015) analysis in Xanthophyllomyces will greatly benefit from associated metabolite analysis in order to evaluate cellular regulation.

The growing interest in Xanthophyllomyces as a potential industrial producer of carotenoids and the importance 


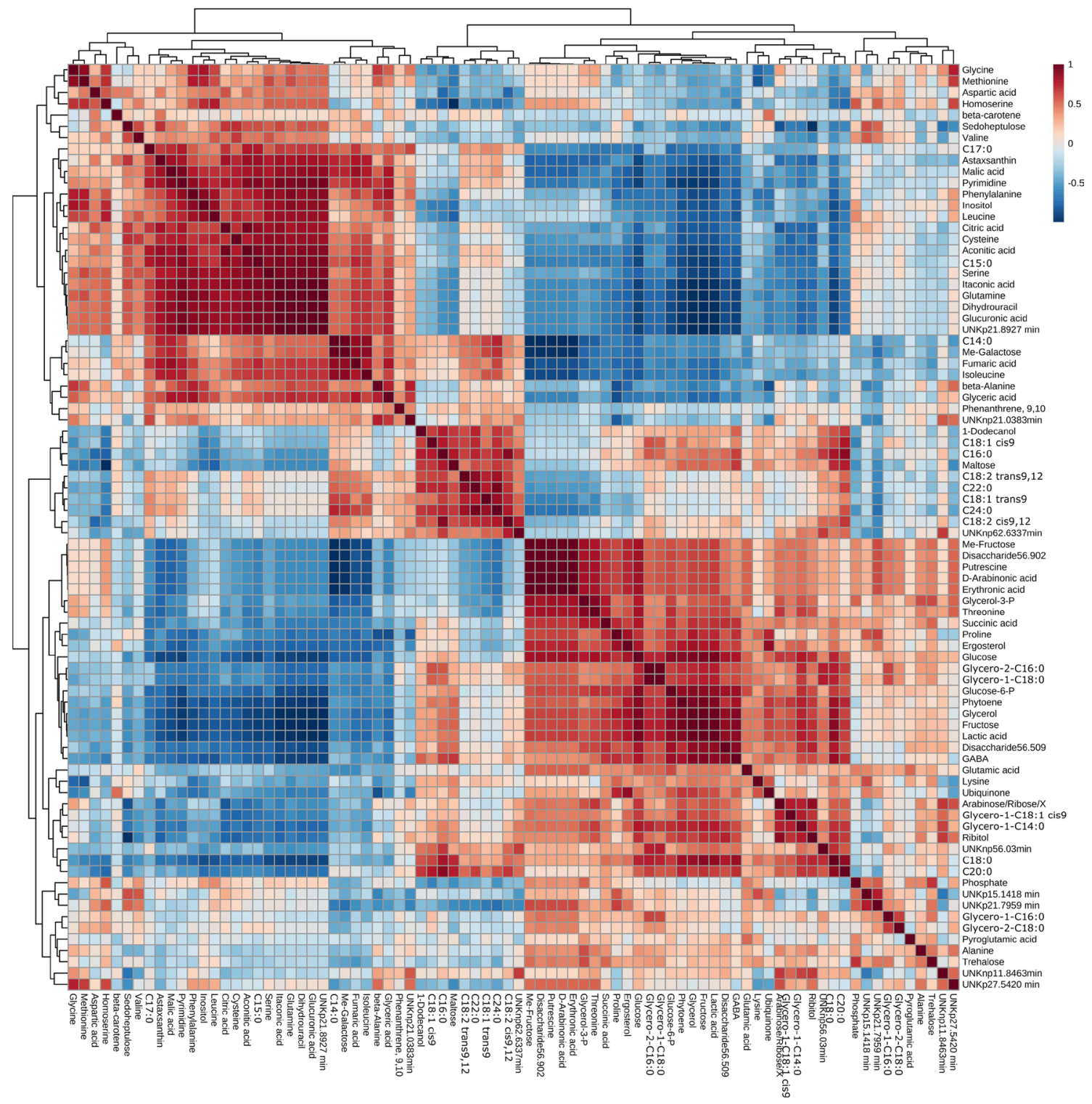

Fig. 7 Heat map of correlation analysis of Xanthophyllomyces metabolites based on Pearson coefficients. The heat map was created using the server MetaboAnalyst and presented in Inkscape

of the "omics" technologies has been recently reported (Barredo et al. 2017). Metabolic engineering and new Synthetic Biology approaches can be applied to enhance specific pathways or to incorporate heterologous ones, which has already demonstrated in Saccharomyces (Meadows et al. 2016) and E. coli (Brunk et al. 2016; Ma et al. 2016; Zhu et al. 2014). The interaction between native biosynthetic pathways and those with the heterologous pathways incorporated is the mayor challenge for Synthetic Biology. The metabolite profiling method presented here provides an important component of the toolkit to approach the challenges of the microbial production of high value metabolites.

\section{Conclusion}

This article reports procedures for the metabolite profiling of the non-conventional industrial yeast Xanthophyllomyces dendrorhous. These procedures have extended our ability to carry out holistic analysis across cellular regulation in this organism. The profiling procedure has been used to determine the relative changes in metabolites 
during it standardised laboratory growth conditions with defined lag, exponential and stationary phases. Interrogation of the data over this cultivation cycle has illustrated that the astaxanthin correlate positively with different sectors of intermediary metabolism (TCA and amino acids), "short-chain" saturated fatty acids and $\beta$-carotene, while other metabolites are diminished in quantity in favour of astaxanthin production; potential providing future targets for the manipulation of metabolism in this organism for the production of valuable compounds that are not directly associated with terpenoid biosynthesis.

\section{Experimental procedures}

\subsection{Xanthophyllomyces strain and culture conditions}

The wild-type Xanthophyllomyces strain CBS 6938 was used in this study. Starting from a pre-culture grown for 2 days and inoculation with a 1:10 ratio with the media. Cultivation was performed in baffled conical flasks $(250 \mathrm{ml})$, containing YPD-medium $(50 \mathrm{ml})$. Following inoculation cultures were incubated for 7 days at $22{ }^{\circ} \mathrm{C}$ in light $\left(16-20 \mu \mathrm{mol} \mathrm{m}^{-2} \mathrm{~s}^{-1}\right)$, while being shaken in an orbital manner at $140 \mathrm{rpm}$.

\subsection{The optimisation of a metabolite profiling procedure for xanthophyllomyces}

In order to evaluate procedures for the quenching of metabolites and then their efficient extraction, Xanthophyllomyces was cultivated in shake culture, harvesting the biomass during the stationary phase. To ensure accurate comparisons could be made the same biomass pool was used during quenching and extraction. Subsequently, cultivation in shake culture was standardised, ensuring designation of lag, exponential and stationary phases through the determination of OD measurements and colony forming units (CFU). Following robust reproducible sample preparation the analytical (GC-MS and UPLC) workflows and data analysis are summarised in (Fig. 8).

\subsection{Quenching the Xanthophyllomyces metabolome}

Different quenching procedures were evaluated. Initially all procedures harvested Xanthophyllomyces biomass by centrifugation of the $50 \mathrm{ml}$ cultures at $3500 \times \mathrm{g}$ for $5 \mathrm{~min}$ at $4{ }^{\circ} \mathrm{C}$ (Eppendorf $5810 \mathrm{R}$, Hamburg, Germany). The remaining pellet was treated as follows: (i) a pre-chilled $\left(-20^{\circ} \mathrm{C}\right)$ quenching solution, composed of $60 \%(\mathrm{v} / \mathrm{v})$ methanol in $0.9 \%$ (w/v) $\mathrm{NaCl}$ totalling $30 \mathrm{ml}$ was added. This approach was termed M60; while (ii) pre-chilled $\left(-20^{\circ} \mathrm{C}\right)$ methanol (30 ml) was designated $\mathrm{M}$; (iii) a wash solution $(30 \mathrm{ml})$, comprised of $0.9 \%(\mathrm{w} / \mathrm{v}) \mathrm{NaCl}$, was termed $(\mathrm{W})$. After centrifugation at $3500 \times g$ for $5 \mathrm{~min}$ at $0{ }^{\circ} \mathrm{C}$, the supernatant was discarded and pelleted cells snap-frozen in liquid nitrogen, then freeze-dried and stored at $-80^{\circ} \mathrm{C}$ until extraction. Nontreated snap-frozen pelleted cells were also used as a control (C).

The different quenching methods were evaluated by monitoring the recovery of four polar-metabolite standards, deuterated $\mathrm{D}_{4}$-succinic acid, $\mathrm{D}_{2}$-glucose, $\mathrm{D}_{5}$-glycine and ribitol, representing an organic acid, monosaccharide, amino acid and polyol chemistries, respectively. These classes of metabolites are rapidly affected by the metabolism. The procedure (Canelas et al. 2009) was used to quantify potential metabolite degradation and/or poor metabolic quenching.

Cultures were grown until stationary phase for optimal biomass. After quenching samples were freeze dried and chloroform:methanol extraction method (CM) was applied for polar extraction. The standards were added to the cells immediately after quenching and before analysis.

\subsection{Extraction of polar metabolites and carotenoids}

The starting material for extraction was standardised, dried biomass $(10 \mathrm{mg})$. The following approaches were tested:

(i) Chloroform:methanol (CM): $50 \%(\mathrm{v} / \mathrm{v})$ methanol $(500 \mu \mathrm{l})$ was added and placed in a sonication bath (Sonicor SC-120 ${ }^{\mathrm{TH}}$, Sonicor Instrument Corporation, Copiague, NY, US) at room temperature. Sonication was performed for $15 \mathrm{~min}$ at $50 / 60 \mathrm{~Hz}$. Chloroform
Fig. 8 Quenching and extraction workflow scheme for Xanthophyllomyces

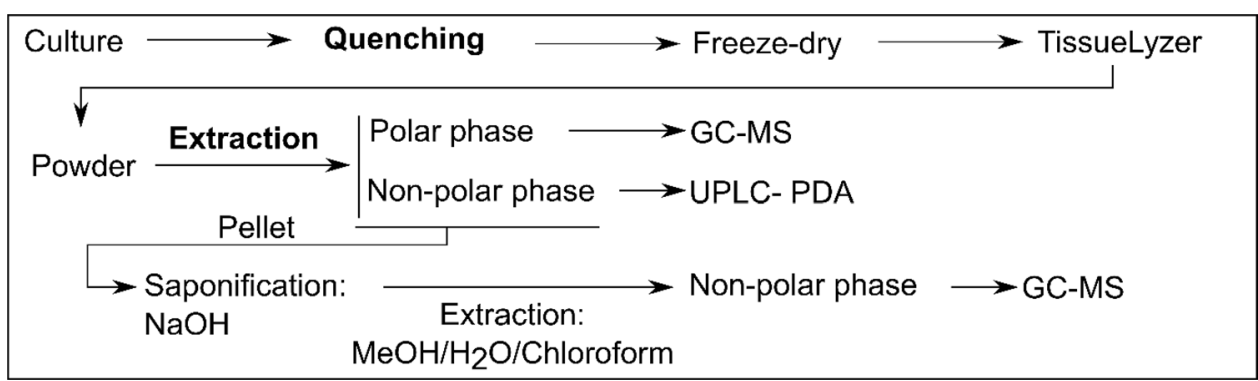


(1 $\mathrm{ml})$ was added and the suspension centrifuged at $14,000 \mathrm{rpm}$ for $5 \mathrm{~min}$ to facilitate phase separation. The epiphase $(500 \mu \mathrm{l})$, containing polar metabolites, was separated from the organic phase (hyperphase) and stored at $-20{ }^{\circ} \mathrm{C}$ until further analysis. The hypophase was stored at $-20{ }^{\circ} \mathrm{C}$ and pooled with the non-polar extracts obtained as described in the following section.

(ii) Chloroform:methanol acidified (CMA): A modification of the chloroform:methanol method was tested by using $50 \%(\mathrm{v} / \mathrm{v})$ methanol $(500 \mu \mathrm{l})$ containing $0.1 \mathrm{M}$ formic acid. The rest of the method proceeded as described for the $\mathrm{CM}$ method.

(iii) $80 \%(\mathrm{v} / \mathrm{v})$ Methanol $(80 \mathrm{M}): 80 \%$ methanol $(1 \mathrm{ml})$ was added and the suspension was sonicated for $15 \mathrm{~min}$. The polar extract was collected after centrifugation $(14,000 \mathrm{rpm}, 5 \mathrm{~min})$ and stored at $-20{ }^{\circ} \mathrm{C}$ prior to analysis.

(iv) Acidified acetonitrile:methanol:water (AAMW): A solution of acetonitrile:methanol:water (40:40:20) (v/v) containing $0.1 \mathrm{M}$ formic acid $(500 \mu \mathrm{l})$ was employed for extracting polar metabolites (Rabinowitz and Kimball 2007). Extracts were then sonicated (15 min) centrifuged (14,000 rpm, $5 \mathrm{~min})$, the polar extracts removed and stored at $-20{ }^{\circ} \mathrm{C}$ prior to analysis.

(v) Boiling ethanol (BEt): $75 \%(\mathrm{v} / \mathrm{v})$ ethanol $(2 \mathrm{ml})$ was added. The suspension was heated at $95{ }^{\circ} \mathrm{C}$ for $3 \mathrm{~min}$ in a water bath and rapidly cooled on ice (Gonzalez et al. 1997; Taymaz-Nikerel et al. 2009). The ethanolic extract was then collected after centrifugation (14,000 rpm, $5 \mathrm{~min})$ and taken to dryness. The sample was dissolved in distilled water $(500 \mu \mathrm{l})$ and an aliquot $(100 \mu \mathrm{l})$ used for analysis.

Cultures were grown until stationary phase for optimal biomass, quenching by washing with $\mathrm{NaCl}$ solution (W), performed and the material pelleted and then freeze-dried to eliminate any remain water and kept at $-80{ }^{\circ} \mathrm{C}$ before the extraction. The five different extraction methods based on different solvent solutions were tested. The same standards applied for the quenching were used. The standards were added to the cells immediately before extraction or prior to analysis (Fig. 1).

\subsection{Extraction of non-polar metabolites}

After extracting polar metabolites the remaining pellets were saponified with aqueous $10 \% \mathrm{NaOH}(\mathrm{w} / \mathrm{v})$ at room temperature and sonicated for $10 \mathrm{~min}$. The $\mathrm{NaOH}$ solution was removed by centrifugation and the pellet was extracted with chloroform:methanol (CM) as described in the previous section e.g. treatment (i). The hypophase, containing non-polar metabolites, was separated from the epiphase and stored at $-20{ }^{\circ} \mathrm{C}$ until further analysis. Phases for polar and non-polar metabolites obtained were dried under vacuum using GENEVAC EZ-2 Plus (GENEVAC LTD., Ipswich, England).

\subsection{Recovery and efficacy}

Metabolite recoveries were calculated according to the following formula (Canelas et al. 2009):

Recovery $_{\mathrm{x} ; \mathrm{i}}=[\mathrm{x}]_{\mathrm{i}} \mathrm{AQ} /[\mathrm{x}]_{\mathrm{i}} \mathrm{BQ}$

where " $x$ " indicates metabolite, " $i$ " stands for the quenching method and the upper indexes BA and AQ meaning that the internal standards (IS) were added before analysis (BA) and after quenching (AQ), respectively. Recovery values much below 1 typically indicate degradation and/or poor quenching of metabolic activity. Similarly, the ability of different extraction methods to release metabolites from biological matrices can be evaluated in terms of efficacy as follows (Canelas et al. 2009):

\section{Efficacy $_{\mathrm{x} ; \mathrm{i}}=[\mathrm{x}]_{\mathrm{i}} \mathrm{BE} /[\mathrm{x}]_{\mathrm{i}} \mathrm{BA}$}

where ' $x$ ', indicates metabolite, " $i$ ', stands for the extraction method and the upper indexes BA and BE meaning that the internal standards were added before analysis (BA) and before extraction (BE), respectively. Aliquots (100 $\mu \mathrm{l})$ of BA samples were spiked with a solution $(20 \mu \mathrm{l})$ of internal standards containing the deuterated standards, Deuterated $\mathrm{D}_{4}$-succinic acid, $\mathrm{D}_{2}$-glucose, $\mathrm{D}_{5}$-glycine and ribitol, at a concentration of $1 \mathrm{mg} / \mathrm{ml}$ each, giving a final amount of $20 \mu \mathrm{g}$ of internal standard in the BA aliquots. The aliquots were then dried under vacuum and derivatised as described in next section. For BE and AQ series the solution of internal standards was prepared at $5 \mathrm{mg} / \mathrm{ml}$ and $20 \mu \mathrm{l}$ used for spiking samples. Since solvent $(500 \mu \mathrm{l})$ was employed for extraction the final concentration of IS was $0.2 \mu \mathrm{g} / \mu \mathrm{l}$ in the polar extract. An aliquot $(100 \mu \mathrm{l})$ was evaporated to give a final amount of $20 \mu \mathrm{g}$ of IS, that was derivatised concurrently with the rest of the components in the extracts as described below.

Dedicated experiments for calculating limit of detection and the limits of quantification were not performed concurrently in the present work as relative quantification (to internal standard) values were used routinely instead of absolute quantification using calibration curves for every metabolite detected. However, generic machine standard calibration curves show linearity in the range of $0.025-0.25 \mu \mathrm{g} / \mu \mathrm{l}$ for monosaccharides and $0.025-0.1 \mu \mathrm{g} /$ $\mu \mathrm{l}$ for disaccharides, and an approximate LOD value can be extrapolated from these calibration curves to be in the range of 6-20 ppm. 


\subsection{GC-MS analysis of polar and non-polar metabolites}

Those polar and non-polar extracts, generated using the methods described in previous sections, were analysed by GC-MS as described in (Alcalde and Fraser 2016) with the following modifications. In brief an aliquot from each polar $(200 \mu \mathrm{l})$ and non-polar $(200 \mu \mathrm{l})$ extract were removed, dried under vacuum and solubilised in the derivatisation reagents. For example, samples were derivatised to their methoxylated and silylated forms according to (Halket et al. 2005). Methoxyamine hydrochloride $(20 \mathrm{mg} / \mathrm{ml}$ in anhydrous pyridine; $30 \mu \mathrm{l}$ ) was added to samples and incubated at $40{ }^{\circ} \mathrm{C}$ for $1 \mathrm{~h}$. Following this reaction, the suspensions were treated with MSTFA $(70 \mu \mathrm{l})$ and heated at $40{ }^{\circ} \mathrm{C}$ for $2 \mathrm{~h}$. The final solution $(1 \mu \mathrm{l})$ was injected in split mode (1:10) into a 7890A GC on-line with a 5975C mass spectrometer (Agilent Technologies, Palo Alto, California, US). Metabolites were separated on a DB-5MS $30 \mathrm{~m} \times 250 \mathrm{~lm} \times 0.25 \mathrm{~lm}$ column (J\&W Scientific, Folsom, California, US), equipped with a $10 \mathrm{~m}$ guard column and using a temperature gradient ranging from 70 to $320^{\circ} \mathrm{C}$ at $5{ }^{\circ} \mathrm{C} \mathrm{min}^{-1}$. Helium was employed as the carrier gas and the flow rate was $0.5 \mathrm{ml} \mathrm{min}^{-1}$. The inlet was heated to $280{ }^{\circ} \mathrm{C}$ and the mass spectrometer transfer line at $250{ }^{\circ} \mathrm{C}$. A mixture of $\mathrm{n}$-alkanes, ranging from 8 to 32 carbons, was used for retention index external calibration. Authentic standards, $\mathrm{D}_{4}$-succinic acid and $\mathrm{D}_{27}$-myristic acid for polar and non-polar extract, respectively, were added at a concentration of $1 \mathrm{mg} / \mathrm{ml}$ and aliquots $(10 \mu \mathrm{l})$ to the samples before dried and subjected to the identical derivatisation procedure as that used with the extracts.

\subsection{Data analysis}

Levels of metabolites analysed by GC-MS were quantified relative to the internal standard and corrected for the dried weight of the biomass. Compounds generating multiple peaks in the chromatogram as a consequence of the methoxylation and silylation reactions were quantified by summing the areas of the different derivatives. AMDIS (version 2.7) software was used for peak deconvolution and establishing the authors' libraries for polar and non-polar metabolites. Identification of metabolites for library construction was done by comparing mass spectra and retention indexes to NIST [version 2.0 (2008)] and Golm Metabolome (http:// gmd.mpimp-golm.mpg.de/analysisinput.aspx) mass spectral databases and confirmed with authentic standards whenever possible. Those compounds not identified were named as UNK followed the sub index $\mathrm{p}$ or $\mathrm{np}$ for polar or non-polar extracts, respectively, and by the corresponding retention time. The identification criteria used and annotation of unknowns was performed as described by Bino et al. (2004) and Sumner et al. (2007). Data matrices were transformed using the pareto-scaled method (van den Berg et al. 2006) and log transformation (Fig. S4), multivariate analysis, time course heat map and correlation analysis performed using the server MetaboAnalyst 3.0 (http://www.metaboanalyst.ca) (Xia et al. 2016). Means, standard deviation, $p$ values and q values (for FDRs, Benjamini and Hochberg 1995) were calculated in Excel. Extraction-method heat map was created with Excel and Inkscape.

\subsection{Separation and detection by UPLC-PDA}

Carotenoids, ergosterol and ubiquinone (Coenzyme Q9) extracted by the method described previously, were separated and identified by Liquid Chromatography with photodiode array detection. An Acquity ultra high performance liquid chromatography system (Waters) was used with an Ethylene Bridged Hybrid (BEH C18) column $(2.1 \times 100 \mathrm{~mm}, 1.7 \mathrm{~mm})$ with a BEH C18 VanGuard precolumn $(2.1 \times 50 \mathrm{~mm}, 1.7 \mathrm{~mm})$. The mobile phase used was $\mathrm{A}$, methanol/water (50/50), and $\mathrm{B}$, acetonitrile (ACN)/ ethyl acetate $(75: 25)$ and the flow rate was $0.5 \mathrm{ml} \mathrm{min}^{-1}$. All solvents used were HPLC grade and filtered prior to use through a $0.2-\mathrm{mm}$ filter. The gradient was $30 \% \mathrm{~A}: 70 \%$ B for $0.5 \mathrm{~min}$ and then stepped to $0.1 \% \mathrm{~A}: 99.9 \% \mathrm{~B}$ for $5.5 \mathrm{~min}$ and then to $30 \%$ A:70\% B for the last 2 min. Column temperature was maintained at $30{ }^{\circ} \mathrm{C}$ and the temperature of samples at $8{ }^{\circ} \mathrm{C}$. Online scanning across the UV/visible range was performed in a continuous manner from 250 to $600 \mathrm{~nm}$, using an extended wavelength photo diode array detector (Waters, Watford, UK).

Acknowledgements We thank Christopher Gerrish for technical support and guidance.

Author contributions Experiments were developed by EA and PDF. All experimental work was performed by EA. All authors contributed to the interpretation of the results and preparation of the manuscript. Original Draft EA; Writing -Review \& Editing, EA and PDF.

Funding This work was funded in part through the ERA-IB PROCAR project, BBSRC BB/MO25829/1 to P.D.F.

\section{Compliance with ethical standards}

Conflict of interest E.A. and P.D.F. declare that they have no conflict of interest.

Ethical approval This article does not contain any studies with human participants or animals performed by any of the authors.

Open Access This article is distributed under the terms of the Creative Commons Attribution 4.0 International License (http://creativeco mmons.org/licenses/by/4.0/), which permits unrestricted use, distribution, and reproduction in any medium, provided you give appropriate credit to the original author(s) and the source, provide a link to the Creative Commons license, and indicate if changes were made. 


\section{References}

Alcaíno, J., Barahona, S., Carmona, M., Lozano, C., Marcoleta, A., Niklitschek, M., et al. (2008). Cloning of the cytochrome p450 reductase (crtR) gene and its involvement in the astaxanthin biosynthesis of Xanthophyllomyces dendrorhous. BMC Microbiology, 8, 169. https://doi.org/10.1186/1471-2180-8-169.

Alcalde, E., \& Fraser, P. D. (2016). Metabolite profiling of Phycomyces blakesleeanus carotene mutants; reveals global changes across intermediary metabolism. Microbiology. https://doi.org/10.1099/ mic. 0.000376

Barbachano-Torres, A., Castelblanco-Matiz, L. M., Ramos-Valdivia, A. C., Cerda-García-Rojas, C. M., Salgado, L. M., Flores-Ortiz, C. M., \& Ponce-Noyola, T. (2014). Analysis of proteomic changes in colored mutants of Xanthophyllomyces dendrorhous (Phaffia rhodozyma). Archives of Microbiology, 196, 411-421. https://doi. org/10.1007/s00203-014-0979-x.

Barredo, J., García-Estrada, C., Kosalkova, K., \& Barreiro, C. (2017). Biosynthesis of astaxanthin as a main carotenoid in the heterobasidiomycetous yeast Xanthophyllomyces dendrorhous. Journal of Fungi, 3(3), 44. https://doi.org/10.3390/jof3030044.

Bellora, N., Moliné, M., David-Palma, M., Coelho, M. A., Hittinger, C. T., Sampaio, J. P., et al. (2016). Comparative genomics provides new insights into the diversity, physiology, and sexuality of the only industrially exploited tremellomycete: Phaffia rhodozyma. BMC Genomics, 17(1), 901. https://doi.org/10.1186/ s12864-016-3244-7.

Benjamini, Y., \& Hochberg, Y. (1995). Controlling the false discovery rate: A practical and powerful approach to multiple testing. Journal of the Royal Statistical Society. Series B (Methodological), 57(1), 289-300. https://doi.org/10.2307/2346101.

Bino, R. J., Hall, R. D., Fiehn, O., Kopka, J., Saito, K., Draper, J., et al. (2004). Potential of metabolomics as a functional genomics tool. Trends in Plant Science, 9(9), 418-425. https://doi.org/10.1016/j. tplants.2004.07.004.

Brunk, E., George, K. W., Alonso-Gutierrez, J., Thompson, M., Baidoo, E., Wang, G., et al. (2016). Characterizing strain variation in engineered E. coli using a multi-omics-based workflow. Cell Systems, 2(5), 335-346. https://doi.org/10.1016/j.cels.2016.04.004.

Business Communications Company. (2015). The global market for carotenoids-FOD025E. Accessed February 7, 2017, from http:// www.bccresearch.com/market-research/food-and-beverage/carot enoids-global-market-report-fod025e.html.

Canelas, A. B., Ten Pierick, A., Ras, C., Seifar, R. M., Van Dam, J. C., Van Gulik, W. M., \& Heijnen, J. J. (2009). Quantitative evaluation of intracellular metabolite extraction techniques for yeast metabolomics. Analytical Chemistry, 81(17), 7379-7389. https ://doi.org/10.1021/ac900999t.

Canelas, A. B., Ras, C., ten Pierick, A., van Dam, J. C., Heijnen, J. J., \& van Gulik, W. M. (2008). Leakage-free rapid quenching technique for yeast metabolomics. Metabolomics, 4(3), 226-239. https://doi. org/10.1007/s11306-008-0116-4

Cataldo, V. F., López, J., Cárcamo, M., \& Agosin, E. (2016). Chemical vs. biotechnological synthesis of C13-apocarotenoids: Current methods, applications and perspectives. Applied Microbiology and Biotechnology, 100(13), 5703-5718. https://doi.org/10.1007/ s00253-016-7583-8.

Drapal, M., Perez-Fons, L., Wheeler, P. R., \& Fraser, P. D. (2014). The application of metabolite profiling to Mycobacterium spp.: Determination of metabolite changes associated with growth. Journal of microbiological methods, 106, 23-32. https://doi.org/10.1016/j. mimet.2014.07.037.

Gessler, N. N., Aver'yanov, A. A., \& Belozerskaya, T. A. (2007). Reactive oxygen species in regulation of fungal development.
Biochemistry, 72(10), 1091-1109. https://doi.org/10.1134/S0006 297907100070.

Gonzalez, B., François, J., \& Renaud, M. (1997). A rapid and reliable method for metabolite extraction in yeast using boiling buffered ethanol. Yeast (Chichester, England), 13(14), 1347-1355. https ://doi.org/10.1002/(SICI)1097-0061(199711)13:14<1347::AIDYEA176>3.0.CO;2-O

Gruszecki, W. I., \& Strzałka, K. (2005). Carotenoids as modulators of lipid membrane physical properties. Biochimica et Biophysica Acta, 1740(2), 108-115. https://doi.org/10.1016/j.bbadi s.2004.11.015.

Halket, J. M., Waterman, D., Przyborowska, A. M., Patel, R. K. P., Fraser, P. D., \& Bramley, P. M. (2005). Chemical derivatization and mass spectral libraries in metabolic profiling by $\mathrm{GC} /$ MS and LC/MS/MS. Journal of Experimental Botany, 56(410), 219-243. https://doi.org/10.1093/jxb/eri069.

Johnson, E. a. (2003). Phaffia rhodozyma: Colorful odyssey. International Microbiology, 6, 169-174. https://doi.org/10.1007/s1012 3-003-0130-3.

Loto, I., Gutiérrez, M. S., Barahona, S., Sepúlveda, D., MartínezMoya, P., Baeza, M., et al. (2012). Enhancement of carotenoid production by disrupting the $\mathrm{C} 22$-sterol desaturase gene (CYP61) in Xanthophyllomyces dendrorhous. BMC Microbiology, 12, 235. https://doi.org/10.1186/1471-2180-12-235.

Lv, H., Xia, F., Liu, M., Cui, X., Wahid, F., \& Jia, S. (2016). Metabolomic profiling of the astaxanthin accumulation process induced by high light in Haematococcus pluvialis. Algal Research, 20, 35-43. https://doi.org/10.1016/j.algal.2016.09.019.

Ma, T., Deng, Z., \& Liu, T. (2016). Microbial production strategies and applications of lycopene and other terpenoids. World Journal of Microbiology \& Biotechnology, 32(1), 15. https://doi. org/10.1007/s11274-015-1975-2.

Mannazzu, I., Landolfo, S., da Silva, T. L., \& Buzzini, P. (2015). Red yeasts and carotenoid production: Outlining a future for nonconventional yeasts of biotechnological interest. World Journal of Microbiology \& Biotechnology, 31(11), 1665-1673. https:// doi.org/10.1007/s11274-015-1927-x.

Martinez-Moya, P., Niehaus, K., Alcaíno, J., Baeza, M., \& Cifuentes, V. (2015). Proteomic and metabolomic analysis of the carotenogenic yeast Xanthophyllomyces dendrorhous using different carbon sources. BMC Genomics, 16(1), 289. https://doi. org/10.1186/s12864-015-1484-6.

Martinez-Moya, P., Watt, S. A., Niehaus, K., Alcaíno, J., Baeza, M., \& Cifuentes, V. (2011). Proteomic analysis of the carotenogenic yeast Xanthophyllomyces dendrorhous. BMC microbiology, 11(1), 131. https://doi.org/10.1186/1471-2180-11-131.

Mashego, M. R., Rumbold, K., De Mey, M., Vandamme, E., Soetaert, W., \& Heijnen, J. J. (2007). Microbial metabolomics: Past, present and future methodologies. Biotechnology Letters, 29(1), 1-16. https://doi.org/10.1007/s10529-006-9218-0.

Meadows, A. L., Hawkins, K. M., Tsegaye, Y., Antipov, E., Kim, Y., Raetz, L., et al. (2016). Rewriting yeast central carbon metabolism for industrial isoprenoid production. Nature, 537(7622), 694-697. https://doi.org/10.1038/nature19769.

Miller, M. W., Yoneyama, M., \& Soneda, M. (1976). Phaffia, a new yeast genus in the Deuteromycotina (Blastomycetes). International Journal of Systematic Bacteriology, 26, 286-291. https ://doi.org/10.1099/00207713-26-2-286.

Niyogi, K. K., Björkman, O., \& Grossman, A. R. (1997). The roles of specific xanthophylls in photoprotection. Proceedings of the National Academy of Sciences of the United States of America, 94(25), 14162-14167. https://doi.org/10.1073/pnas.94.25.14162.

Ojima, K., Breitenbach, J., Visser, H., Setoguchi, Y., Tabata, K., Hoshino, T., et al. (2006). Cloning of the astaxanthin synthase gene from Xanthophyllomyces dendrorhous (Phaffia rhodozyma) and its assignment as a beta-carotene 3-hydroxylase/4-ketolase. 
Molecular Genetics and Genomics: MGG, 275(2), 148-158. https ://doi.org/10.1007/s00438-005-0072-x.

Perez-Fons, L., Bramley, P. M., \& Fraser, P. D. (2014). The optimisation and application of a metabolite profiling procedure for the metabolic phenotyping of Bacillus species. Metabolomics, 10(1), 77-90. https://doi.org/10.1007/s11306-013-0553-6.

Phaff, H. J., Miller, M. W., Yoneyama, M., \& Soneda, M. (1972). A comparative study of the yeast florae associated with trees on the Japanese Islands and on the west coast of North America. In G. Terui (Ed.), Fourth international fermentation symposium proceedings: Fermentation technology today (pp. 759-774). Osaka, Japan: Society of Fermentation Technology.

Prasad Maharjan, R., \& Ferenci, T. (2003). Global metabolite analysis: The influence of extraction methodology on metabolome profiles of Escherichia coli. Analytical Biochemistry, 313(1), 145-154. https://doi.org/10.1016/S0003-2697(02)00536-5.

Rabinowitz, J. D., \& Kimball, E. (2007). Acidic acetonitrile for cellular metabolome extraction from Escherichia coli. Analytical Chemistry, 79(16), 6167-6173. https://doi.org/10.1021/ac070470c.

Schmidt, I., Schewe, H., Gassel, S., Jin, C., Buckingham, J., Hümbelin, M., et al. (2011). Biotechnological production of astaxanthin with Phaffia rhodozyma/Xanthophyllomyces dendrorhous. Applied Microbiology and Biotechnology, 89, 555-571. https:// doi.org/10.1007/s00253-010-2976-6.

Schroeder, W. A., \& Johnson, E. A. (1993). Antioxidant role of carotenoids in Phaffia rhodozyma. Journal of General Microbiology, 139(5), 907-912. https://doi.org/10.1099/00221287-139-5-907.

Sharma, R., Gassel, S., Steiger, S., Xia, X., Bauer, R., Sandmann, G., \& Thines, M. (2015). The genome of the basal agaricomycete Xanthophyllomyces dendrorhous provides insights into the organization of its acetyl-CoA derived pathways and the evolution of Agaricomycotina. BMC Genomics, 16(1), 233. https://doi. org/10.1186/s12864-015-1380-0.

Sumner, L. W., Amberg, A., Barrett, D., Beale, M. H., Beger, R., Daykin, C. A., et al. (2007). Proposed minimum reporting standards for chemical analysis. Metabolomics, 3(3), 211-221. https:// doi.org/10.1007/s11306-007-0082-2.

Takagi, H. (2008). Proline as a stress protectant in yeast: Physiological functions, metabolic regulations, and biotechnological applications. Applied Microbiology and Biotechnology. https://doi. org/10.1007/s00253-008-1698-5.

Taymaz-Nikerel, H., de Mey, M., Ras, C., ten Pierick, A., Seifar, R. M., van Dam, J. C., et al. (2009). Development and application of a differential method for reliable metabolome analysis in Escherichia coli. Analytical Biochemistry, 386(1), 9-19. https:// doi.org/10.1016/j.ab.2008.11.018.

van den Berg, R. A., Hoefsloot, H. C. J., Westerhuis, J. A., Smilde, A. K., \& van der Werf, M. J. (2006). Centering, scaling, and transformations: Improving the biological information content of metabolomics data. BMC Genomics, 7(1), 142. https://doi. org/10.1186/1471-2164-7-142.

Van Urk, H., Postma, E., Scheffers, A. W., \& Van Dijken, J. P. (1989). Glucose transport in Carbtree-postive and Carbtree negative yeasts. Microbiology, 135, 2399-2406.

Verdoes, J. C., Krubasik, K. P., Sandmann, G., \& van Ooyen, A. J. (1999). Isolation and functional characterisation of a novel type of carotenoid biosynthetic gene from Xanthophyllomyces dendrorhous. Molecular \& General Genetics: MGG, 262(3), 45361. Accessed April 18, 2016, from http://www.ncbi.nlm.nih.gov/ pubmed/10589832.

Verdoes, J. C., Misawa, N., \& Van Ooyen, A. J. J. (1999). Cloning and characterization of the astaxanthin biosynthetic gene encoding phytoene desaturase of Xanthophyllomyces dendrorhous. Biotechnology and Bioengineering, 63(6), 750-755. https://doi. org/10.1002/(SICI)1097-0290(19990620)63:6<750::AID-BIT13 $>3.0 . \mathrm{CO} ; 2-7$.

Verdoes, J. C., Sandmann, G., Visser, H., Diaz, M., van Mossel, M., \& van Ooyen, A.J. J. (2003). Metabolic engineering of the carotenoid biosynthetic pathway in the yeast Xanthophyllomyces dendrorhous (Phaffia rhodozyma). Applied and Environmental Microbiology, 69(7), 3728-38. Accessed April 18, 2016, from http:// www.pubmedcentral.nih.gov/articlerender.fcgi?artid=16515 0\&tool $=$ pmcentrez\&rendertype $=$ abstract.

Villas-Bôas, S. G., Højer-Pedersen, J., Åkesson, M., Smedsgaard, J., \& Nielsen, J. (2005). Global metabolite analysis of yeast: Evaluation of sample preparation methods. Yeast, 22(14), 1155-1169. https ://doi.org/10.1002/yea.1308.

Villas-Bôas, S. G., Mas, S., Åkesson, M., Smedsgaard, J., \& Nielsen, J. (2005). Mass spectrometry in metabolome analysis. Mass Spectrometry Reviews, 24(5), 613-646. https://doi.org/10.1002/ mas.20032.

Winder, C. L., Dunn, W. B., Schuler, S., Broadhurst, D., Jarvis, R., Stephens, G. M., \& Goodacre, R. (2008). Global metabolic profiling of Escherichia coli cultures: An evaluation of methods for quenching and extraction of intracellular metabolites. Analytical Chemistry, 80(8), 2939-2948. https://doi.org/10.1021/ac7023409.

Xia, J., Wishart, D. S., Xia, J., \& Wishart, D. S. (2016). Using MetaboAnalyst 3.0 for comprehensive metabolomics data analysis. In Current protocols in bioinformatics (pp. 14.10.1-14.10.91). Hoboken, NJ: Wiley.

Zhu, F., Zhong, X., Hu, M., Lu, L., Deng, Z., \& Liu, T. (2014). In vitro reconstitution of mevalonate pathway and targeted engineering of farnesene overproduction in Escherichia coli. Biotechnology and Bioengineering, 111(7), 1396-1405. https://doi.org/10.1002/ bit.25198. 